\title{
The role of preoperative ureteral stenting in retrograde intrarenal surgery in renal stone patients: a propensity score-matched study
}

\author{
Luck Hee Sung, Dae Yeon Cho \\ Department of Urology, Inje University Sanggye Paik Hospital, Seoul, Korea \\ Contributions: (I) Conception and design: All authors; (II) Administrative support: None; (III) Provision of study materials or patients: None; (IV) \\ Collection and assembly of data: All authors; (V) Data analysis and interpretation: DY Cho; (VI) Manuscript writing: All authors; (VII) Final approval \\ of manuscript: All authors. \\ Correspondence to: Dae Yeon Cho, MD. Department of Urology, Inje University Sanggye Paik Hospital, 1342 Dongil-ro, Nowon-gu, Seoul 01757, \\ Korea. Email: s5524@paik.ac.kr; waytogo@naver.com.
}

\begin{abstract}
Background: The purpose of this study was to investigate the effect of preoperative ureteral stenting on retrograde intrarenal surgery (RIRS).

Methods: We retrospectively analyzed RIRS cases from October 2014 to June 2017. Patients were divided into two groups according to preoperative ureteral stent insertion. The characteristics of the patients [age, body mass index (BMI), sex, previous disease history, hemoglobin, creatinine, estimated glomerular filtration rate (eGFR)], characters of stone (size, number, density, location), ureteral access sheath (UAS) success rate, perioperative complication, operative time, hospitalization time, period with stent, postoperative urinary tract infection (UTI) rate, stone free rate (SFR), and additional treatment rate were analyzed.

Results: RIRS was performed for 122 patients. Seventy-three patients had preoperative ureteral stents before RIRS, while 49 patients did not have preoperative ureteral stents. The median size of the stone was $14.5 \mathrm{~mm}$. Overall SFR was $87.7 \%$. Preoperative eGFR was relatively high in patients who underwent preoperative stenting (68.18 vs. $\left.79.01 \mathrm{~mL} / \mathrm{min} / 1.73 \mathrm{~m}^{2}, \mathrm{P}=0.042\right)$. Preoperative stenting led to improvement in the success rate of UAS insertion $(97.3 \%$ vs. $87.8 \%, \mathrm{P}=0.038)$ during surgery. Before and after propensity score matching revealed a significant difference in operation time when the diameter of the stone was smaller than $1 \mathrm{~cm}(\mathrm{P}=0.019$ and $\mathrm{P}=0.004)$. However, there was no significant difference in operation time, postoperative UTI rate, additional treatment rate, or SFR.
\end{abstract}

Conclusions: Preoperative ureteral stenting can facilitate UAS insertion, and reduce operation time in RIRS for stones with a diameter less than $1 \mathrm{~cm}$.

Keywords: Kidney; renal stone; retrograde intrarenal renal surgery; stent; ureter

Submitted Nov 13, 2019. Accepted for publication Feb 14, 2020.

doi: $10.21037 /$ tau.2020.03.09

View this article at: http://dx.doi.org/10.21037/tau.2020.03.09

\section{Introduction}

Retrograde intrarenal surgery (RIRS) is one of the treatments considered primarily for kidney stone with diameter less than $2 \mathrm{~cm}$ (1). Ureteral access sheath (UAS) is an essential and commonly used tool for RIRS (2). The benefit of the use of UAS in RIRS has been proven in various studies. UAS is advantageous as it facilitates repeated intra-ureteral insertion of the flexible ureteroscope and allows irrigation fluid to be increased while maintaining low pressure in the kidneys, thus facilitating perfusion and vision during endoscopic surgery (3-5).

However, sometimes the sheath does not fit well, causing difficulty in operation. For ureter with a very small diameter, UAS insertion might not be possible. It has been reported that up to $10 \%$ of patients are unable to insert UAS because of the diameter of a small ureter or a small orifice, because of their small ureteral diameter (6). To avoid failure, a variety of methods have been used to enable UAS insertion 
when performing RIRS (6). One such method is a manual dilation of the ureter via pre-operative stent insertion and the method is considered as effective and safe (7). However, as the operation is performed in two stages, there exist possibilities of increase in patient's discomfort and cost of surgery (6). Several studies have reported that preoperative ureteral stenting affects the outcome of ureteroscopic stone surgery (8-10). Some authors have also argued that the preoperative ureteral stent can extend the ureter passively (7). However, none of the studies has reported the relationship between preoperative ureteral stenting and success rate of accessed sheath insertion. Therefore, the objective of this study was to investigate the relationship between preoperative ureteral stenting and UAS insertion and analyze the effect of renal stone surgery on outcome.

\section{Methods}

The study was approved by the Institutional Review Board of Inje Universisy Sanggye Paik Hospital (approval code: H-2018-10-003). This was a retrospective study and exempted from patient written consent. All contents and protocols related to this study followed the Helsinki guidelines. The primary endpoint was the stone free rates (SFRs) between the ureter and preoperative groups. And the secondary endpoint was related to the operation duration, complication rates, access sheath insertion success rates, and clinical SFRs (less than $4 \mathrm{~mm}$ ). The inclusion criteria were those age 18 or older who underwent RIRS surgery with renal stones October 2014-June 2017. Only those cases wherein UAS was performed during the RIRS surgery were included. Some patients had delayed RIRS surgery after the ureteral stent was inserted because the ureteroscopic lithotripsy (URS) was not inserted. Some patients had a pre-stenting plan before surgery. Some patients have had surgery for renal stones after the ureteral stent was inserted for a variety of reasons, including pain, acute kidney injury, infection, and hydronephrosis. Medical records of 122 patients who underwent RIRS with renal stone from October 2014 to June 2017 in a single institutional hospital were retrospectively analyzed. Patients who underwent previous stone surgery, such as RIRS, URS, percutaneous nephrolithotomy (PCNL), minimally invasive PCNL, or laparoscopic ureterolithotomy were excluded. Patients who underwent ureter surgery such as pyeloplasty and those who had ureteral stricture, were also excluded. Eligible patients were divided into prestented group and non-stented group. Preoperative ureteral stent insertion was performed under local anesthesia. First, retrograde pyelography using cystoscopy and the open-ended $5-\mathrm{Fr}$ ureteral catheter was performed and the ureteral stent was inserted using hydrophilic and stiff guide wire. The size of the ureteral stent inserted before surgery was 6-7 Fr. The preoperative ureteral stent indwelling period was 1-2 weeks. RIRS was usually performed about $1-2$ weeks subsequent to stent insertion. All patients, except those with urine culture positive, generally administered thirdgeneration cephalosporin (ceftriaxone $2 \mathrm{~g}$ ) one hour before surgery. In the case of symptomatic systemic infection or urine culture positive, antibiotics were used for 1-2 weeks. After confirming that the culture was negative, surgery was performed. All patients underwent surgery under general anesthesia. UAS insertion was attempted prior to the flexible ureteroscopy procedure. A flexible ureteroscope was inserted under a hydrophilic guide wire for patients with narrow ureter and in whom UAS insertion was a failure. The size of the UAS was mostly $12 / 14$ Fr. In some cases, it was $10 / 12 \mathrm{Fr}$ or $11 / 13 \mathrm{Fr}$. UAS insertion routinely tries $12 / 14 \mathrm{Fr}$ UAS first. If the 12/14 Fr UAS does not fit, try the 11/13 Fr and 10/12 Fr with smaller inner diameters. The average length was $45 \mathrm{~cm}$ for men and $35 \mathrm{~cm}$ for women. Stone fragmentation was performed using holmium: yttrium-aluminum-garnet (Holmium YAG) laser. Removal of the stone was done using a stone basket to the maximal extent possible. Dusting was performed on the remaining residual stone. Ureter injury was confirmed by examining the inside of the ureter when removing the UAS, accompanied with the flexible URS, and removing the UAS and descending from the renal pelvis to the distal ureter. If there were no postoperative complications such as a ureter injury, the postoperative ureter stent was removed one week after the operation routinely. SFR was defined as the diameter of the residual stones on CT, 0 and $<4 \mathrm{~mm}$. CT was generally performed three months after RIRS.

The characteristics of the patients [age, body mass index (BMI), sex, previous disease, hemoglobin, creatinine (Cr), estimated glomerular filtration rate (eGFR)], characters of stone (location, size, number, density, laterality), success rate of UAS insertion, intraoperative complication, operative time, hospitalization time, period with ureteral stent, postoperative urinary tract infection (UTI) rate, SFR, and additional treatment rate were analyzed. The difference in operation time according to stone size was also analyzed.

In statistical analysis, continuous variables were expressed as median value and range or mean values and standard 
deviation using technical statistics. Nominal variables are expressed as a probability (\%) through frequency analysis and crossover analysis. The primary endpoint was SFR analyzed based on two criteria: the complete free rate of $0 \mathrm{~mm}$ and a clinical free rate of less than $4 \mathrm{~mm}$. The secondary endpoint was the difference in operation time and success rate of access sheath insertion according to the use of a preoperative ureteral stent. One-to-one propensity score-matched analysis was performed. The propensity score was calculated by preoperative covariates using multivariate regression analysis for each patient. Covariates were age, BMI, sex, Cr level, eGFR, hydronephrosis, and stone characteristics. All statistical tests were performed using IBM SPSS Statistics, version 22.0 (IBM, Armonk, NY, USA) and a $\mathrm{P}$ value of less than 0.05 was considered statistically significant.

\section{Results}

A total of 122 patients were investigated. The nonstented group comprised of 49 patients and the prestented group comprised of 73 patients. The mean age of the patients was $54.6 \pm 14$.6 years in the non-stented group and $56.7 \pm 14.0$ years in the prestented group. There were no significant differences in age $(\mathrm{P}=0.440)$, gender $(\mathrm{P}=0.579)$, diabetes mellitus $(\mathrm{P}=0.914)$, hypertension $(\mathrm{P}=0.239)$, cardiovascular disease $(\mathrm{P}=0.707)$, cerebrovascular event $(\mathrm{P}=0.348)$, preoperative $\mathrm{Cr}$ level $(\mathrm{P}=0.966)$, or preoperative hemoglobin level $(\mathrm{P}=0.570)$ between the two groups. However, the value of eGFR in the non-stented group was significantly lower compared with the prestented group $(68.18 \pm 32.87$ vs. $79.01 \pm 25.12, \mathrm{P}=0.042)$. In the prestented group, mean $\mathrm{Cr}$ level was $1.4 \mathrm{mg} / \mathrm{dL}$ and mean eGFR was $55.31 \mathrm{~mL} / \mathrm{min} / 1.73 \mathrm{~m}^{2}$ at the time of preoperative ureteral stent indwelling. There were no significant differences in stone characteristics such as laterality $(\mathrm{P}=0.461)$, density $(\mathrm{P}=0.262)$, size $(\mathrm{P}=0.662)$, number $(\mathrm{P}=0.206)$, or accompanied hydronephrosis $(\mathrm{P}=0.424)$ between the two groups (Table 1).

Intraoperative ureter injury and bleeding were not associated with preoperative ureteral stent indwelling $(\mathrm{P}=0.151)$. There were $4(5.5 \%)$ intraoperative complications in the prestented group, including one intrarenal bleeding, three ureteral injury. For the intrarenal bleeding case, intraoperative transfusion was performed. However, no additional procedures such as embolization were required. Postoperative UTI occurred in 3 (6.1\%) cases in the nonstented group and in six cases $(8.2 \%)$ in the prestented group $(\mathrm{P}=0.664)$. As all eight cases of postoperative UTI showed abnormal urine test and more than 38 degrees of fever, they were treated with antibiotics. One case was sepsis requiring intensive care unit treatment. There was no significant difference in intraoperative ureter injury and bleeding $(\mathrm{P}=0.151$ and 0.411$)$, postoperative UTI $(\mathrm{P}=0.664)$, SFR $(\mathrm{P}=0.700)$, clinically $\mathrm{SFR}<4 \mathrm{~mm}(\mathrm{P}=0.827)$, operative time $(\mathrm{P}=0.664)$, additional treatment $(\mathrm{P}=0.991)$, hospitalization period ( $\mathrm{P}=0.678)$, or postoperative period with stent $(\mathrm{P}=0.471)$ between the two groups. There was no significant difference in intraoperative and postoperative complications between the two groups after propensity score matching (Table 2). However, a significant difference in the success rate of UAS insertion according to the presence of preoperative ureter stent insertion was observed. The success rate of UAS insertion was achieved in $87.8 \%$ in the non-stented group and $97.3 \%$ in the prestented group $(\mathrm{P}=0.038)$. The success rate of UAS insertion was also significantly different between the two groups after propensity score matching $(\mathrm{P}=0.035)$ (Table 2). The perioperative complications were not significantly different between the two groups (Table 3).

Operation time was $65.2 \pm 46.4$ minutes in the prestented group and $68.8 \pm 40.8$ minutes in the non-stented group, demonstrating no significant difference between the two groups. The stone size was divided into $1 \mathrm{~cm}$ intervals. For the patients in the prestented group with a stone size of $1 \mathrm{~cm}$ or less, operation time was $40.3 \pm 23.3$ minutes, which was significantly shorter compared with the patients in the nonstented group (48.6 \pm 24.5 minutes). However, there was no significant difference in operative time between the two groups with a stone size of more than $1 \mathrm{~cm}$ (Table 4). Even though the age, BMI, sex, Cr level, eGFR, hydronephrosis, and stone characteristics were corrected through propensity matching (Table 1), the operation time in patients with the stone size of more than $1 \mathrm{~cm}$ was shorter in the prestented group compared with the non-prestented group (Table 4).

\section{Discussion}

Flexible RIRS procedure was first introduced by Bagley et al. in 1987 (11). Since then, the development of optical technology, the advancement of surgical methods and instruments, and expansion of RIRS have led to an increase in the role of RIRS in primary treatment for kidney stones with a diameter less than $2 \mathrm{~cm}(1,7,12,13)$. One of the advanced tools is UAS. UAS in RIRS allows rapid and repeated entry into the ureter and kidney. It also prevents 
Table 1 Patients characteristics according to preoperative ureteral stenting of patients before and after propensity score matching

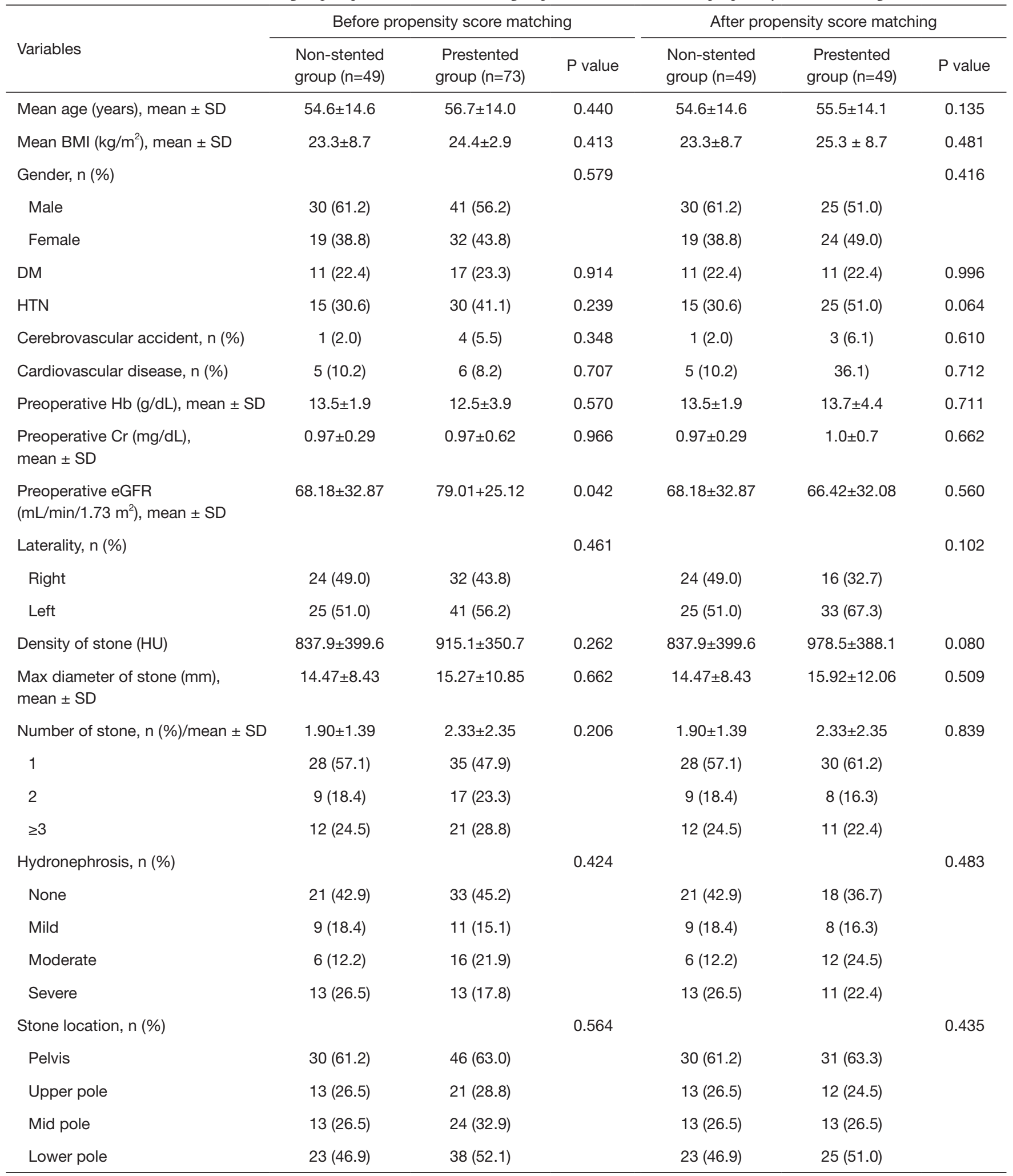

Table 1 (continued) 
Table 1 (continued)

\begin{tabular}{|c|c|c|c|c|c|c|}
\hline Variables & \multicolumn{3}{|c|}{ Before propensity score matching } & \multicolumn{3}{|c|}{ After propensity score matching } \\
\hline Calcium oxalate monohydrate & $34(69.4)$ & $45(61.6)$ & & $34(69.4)$ & $34(69.4)$ & \\
\hline Uric acid & $11(22.4)$ & $20(27.4)$ & & $11(22.4)$ & $12(24.5)$ & \\
\hline Struvite & $1(2.0)$ & $1(1.4)$ & & $1(2.0)$ & $1(2.0)$ & \\
\hline
\end{tabular}

SD, standard deviation; BMI, body mass index; Cr, creatinine; DM, diabetes mellitus; eGFR, estimated glomerular filtration rate; Hb, hemoglobin; HTN, hypertension; HU, Hounsfield units.

Table 2 Perioperative outcomes according to preoperative ureteral stenting before and after propensity score matching

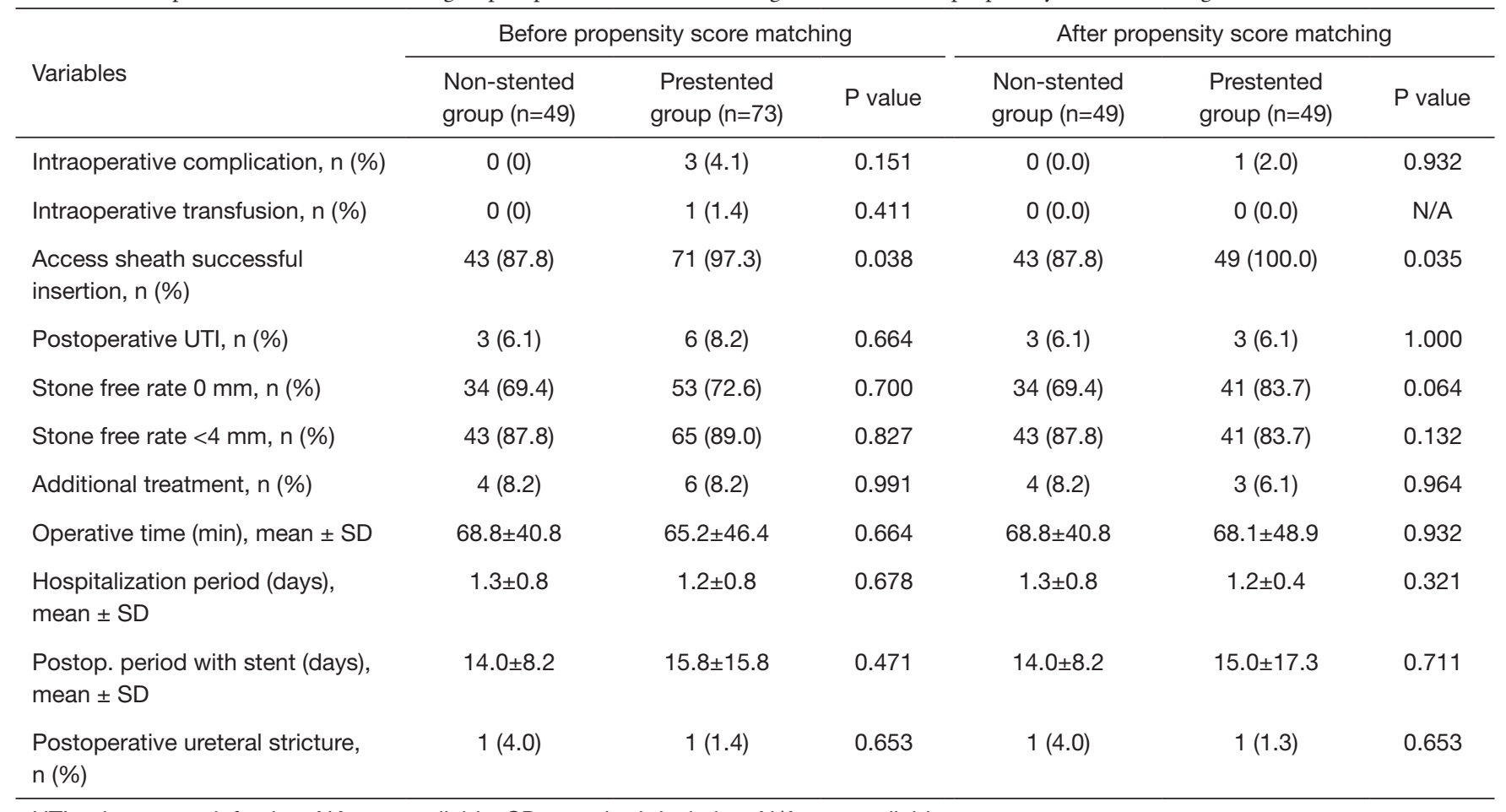

UTI, urinary tract infection; NA, not available; SD, standard deviation; N/A, not available.

pyelovenous backflow during surgery and maintains low renal pressure, thus lowering renal injury while providing a better view (4,14-17). Despite the usefulness of UAS, we cannot insert UAS into the ureter during every surgery. In previous studies, the failure probability of UAS insertion varied from $3 \%$ to $22 \%(9,18-20)$. The causes of failure of UAS insertion is usually because of narrow ureter orifices or narrow ureter diameters relative to the diameter of the UAS. The diameter of the native ureter is usually 6-9 Fr, and the diameter of the outer sheath of a commonly used UAS is $11-14 \operatorname{Fr}(21)$. In the case of RIRS without UAS, the renal pelvis pressure increases, and the increased pressure forms a pyelovenous backflow. The increased pyelovenous backflow leads to renal damage or increased infection 
Table 3 Perioperative complication according to Clavien-Dindo classification

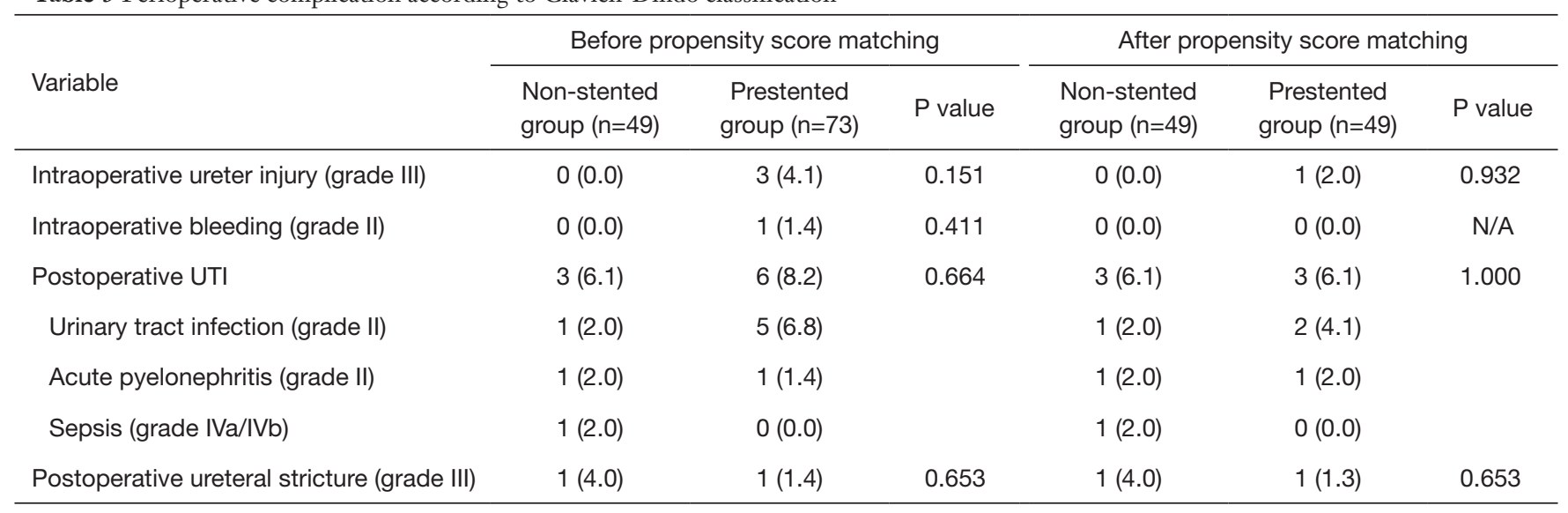

UTI, urinary tract infection.

Table 4 Operation time according to stone size in propensity matching

\begin{tabular}{|c|c|c|c|c|c|c|}
\hline $\begin{array}{l}\text { Size of } \\
\text { stone }(\mathrm{cm})\end{array}$ & \multicolumn{3}{|c|}{ Before propensity score matching, mean \pm SD } & \multicolumn{3}{|c|}{ After propensity score matching, mean $\pm S D$} \\
\hline $1-2$ & $67.6 \pm 34.2$ & $64.2 \pm 48.4$ & 0.777 & $67.6 \pm 34.2$ & $70.6 \pm 49.6$ & 0.588 \\
\hline
\end{tabular}

$\mathrm{SD}$, standard deviation.

risk. As such, UAS insertion may be closely related to postoperative complications (22).

In the present study, the success rate of UAS insertion was significantly higher in the prestented group (97.3\%) compared to the non-stented group $(87.8 \%)$ with $\mathrm{P}=0.038$. The indwelling preoperative ureteral stent showed a significant difference in success rates of UAS insertion. Mogilevkin et al. reported that the prestented ureteral catheter was effective for UAS insertion and was an independent predictor of UAS insertion in multivariate analysis (23). The manually inserted preoperative ureteral stent enlarges the ureter and ureter orifice, corrects the kinking, and corrects the position of the ureter. The changes in the ureter help to increase the success rate of UAS insertion (20-22).

In the present study, the indwelling preoperative ureteral stent shortened the operation time for patients with stone sizes $\leq 1 \mathrm{~cm}$. Stones less than $1 \mathrm{~cm}$ were $25.9 \%$ in the prestented group and $27.1 \%$ in the non-stented group. Stones less than $8 \mathrm{~mm}$ were $16.5 \%$ in the prestented group and $17.6 \%$ in the non-stented group. Stones less than $4 \mathrm{~mm}$ were $1 \%$ in the prestented group and $3.5 \%$ in the nonstented group. There was no significant difference between the two groups. The prestented ureteral stent could extend the ureteropelvic junction and dilate the ureter, enabling easier access, basket stone retrieval and better view (24). A UAS can be easily inserted in the case of prestented patients. And it is sensible that smaller stones could be easily removed from a dilated ureter. In patients with stones less than $1 \mathrm{~cm}$, fragmentation was performed in large pieces rather than dusting, and the number of stones retrieved was smaller compared to relatively large stones, and the field of view after fragmentation was good, which could have affected the operation time. In previous studies, there was no significant difference in operation time between with and without preoperative stents in patients with kidney stones $(9,18-20)$. A few studies reported the difference in operation time according to preoperative stent placement (10). On the contrary, Chu et al. reported that preoperative ureter stent insertion can significantly reduce operative time in patients with stones greater than $1 \mathrm{~cm}$ (10). The difference in stone burden, the difference in diameter of the access sheath, 
and the method of stone fragmentation were not corrected between these studies.

A significant difference in preoperative eGFR was observed $(\mathrm{P}=0.042)$. The value of eGFR was 68.18 $\pm 32.87 \mathrm{~mL} / \mathrm{min} / 1.73 \mathrm{~m}^{2}$ in the non-stented group and $79.01 \pm 25.12 \mathrm{~mL} / \mathrm{min} / 1.73 \mathrm{~m}^{2}$ in the prestented group. It is hypothesized that the ureter stent might have solved the obstruction caused by renal stone and upper ureter stone. Several studies have reported that preoperative ureteral stent indwelling can affect the outcome of RIRS $(25,26)$.

This study has some limitations. First, it was a retrospective study. Second, we did not perform random preoperative stent insertion. Therefore, there is a possibility of the presence of selection bias. However, preoperative ureter catheter insertion is more likely to be performed for difficult or symptomatic cases. It is believed that correction of adverse conditions would generate more significant results. Thirds although propensity matching was conducted, the number of patients was limited, and the power of the conclusions obtained may be insufficient.

\section{Conclusions}

Our results showed that preoperative ureteral stenting can reduce operation time in RIRS for stones with diameters less than $1 \mathrm{~cm}$. Apparently preoperative ureteral insertion can be considered for surgery requiring successful placement of the UAS.

\section{Acknowledgments}

Funding: This work was supported by the 2010 Inje University research grant.

\section{Footnote}

Conflicts of Interest: All authors have completed the ICMJE uniform disclosure form (available at http://dx.doi. org/10.21037/tau.2020.03.09). The authors have no conflicts of interest to declare.

Ethical Statement: The authors are accountable for all aspects of the work in ensuring that questions related to the accuracy or integrity of any part of the work are appropriately investigated and resolved. The study was approved by the Institutional Review Board of Inje University Sanggye Paik Hospital (approval code: H-2018-
10-003). This was a retrospective study and exempted from patient written consent.

Open Access Statement: This is an Open Access article distributed in accordance with the Creative Commons Attribution-NonCommercial-NoDerivs 4.0 International License (CC BY-NC-ND 4.0), which permits the noncommercial replication and distribution of the article with the strict proviso that no changes or edits are made and the original work is properly cited (including links to both the formal publication through the relevant DOI and the license). See: https://creativecommons.org/licenses/by-nc$\mathrm{nd} / 4.0 /$.

\section{References}

1. Bultitude M, Smith D, Thomas K. Contemporary Management of Stone Disease: The New EAU Urolithiasis Guidelines for 2015. Eur Urol 2016;69:483-4.

2. Breda A, Emiliani E, Millan F, et al. The new concept of ureteral access sheath with guidewire disengagement: One wire does it all. World J Urol 2016;34:603-6.

3. Auge BK, Pietrow PK, Lallas CD, et al. Ureteral access sheath provides protection against elevated renal pressures during routine flexible ureteroscopic stone manipulation. J Endourol 2004;18:33-6.

4. Rehman J, Monga M, Landman J, et al. Characterization of intrapelvic pressure during ureteropyeloscopy with ureteral access sheaths. Urology 2003;61:713-8.

5. Vanlangendonck R, Landman J. Ureteral access strategies: pro-access sheath. Urol Clin North Am 2004;31:71-81.

6. Bourdoumis A, Tanabalan C, Goyal A, et al. The difficult ureter: stent and come back or balloon dilate and proceed with ureteroscopy? What does the evidence say? Urology 2014;83:1-3.

7. Miernik A, Wilhelm K, Ardelt PU, et al. Standardized flexible ureteroscopic technique to improve stone-free rates. Urology 2012;80:1198-202.

8. Lumma PP, Schneider P, Strauss A, et al. Impact of ureteral stenting prior to ureterorenoscopy on stone-free rates and complications. World J Urol 2013;31:855-9.

9. Kawahara T, Ito H, Terao H, et al. Preoperative stenting for ureteroscopic lithotripsy for a large renal stone. Int J Urol 2012;19:881-5.

10. Chu L, Sternberg KM, Averch TD. Preoperative stenting decreases operative time and reoperative rates of ureteroscopy. J Endourol 2011;25:751-4. 
11. Bagley DH, Huffman JL, Lyon ES. Flexible ureteropyeloscopy: diagnosis and treatment in the upper urinary tract. J Urol 1987;138:280-5.

12. Wang AJ, Preminger GM. Modern applications of ureteroscopy for intrarenal stone disease. Curr Opin Urol 2011;21:141-4.

13. Desai MR, Ganpule A. Flexible ureterorenoscopy. BJU Int 2011;108:462-74.

14. L'esperance JO, Ekeruo WO, Scales CD Jr, et al. Effect of ureteral access sheath on stone-free rates in patients undergoing ureteroscopic management of renal calculi. Urology 2005;66:252-5.

15. Ng YH, Somani BK, Dennison A, et al. Irrigant flow and intrarenal pressure during flexible ureteroscopy: the effect of different access sheaths, working channel instruments, and hydrostatic pressure. J Endourol 2010;24:1915-20.

16. Stern JM, Yiee J, Park S. Safety and efficacy of ureteral access sheaths. J Endourol 2007;21:119-23.

17. Monga M, Bodie J, Ercole B. Is there a role for smalldiameter ureteral access sheaths? Impact on irrigant flow and intrapelvic pressures. Urology 2004;64:439-41; discussion 441-2.

18. Zhang J, Xu C, He D, et al. Flexible ureteroscopy for renal stone without preoperative ureteral stenting shows good prognosis. PeerJ 2016;4:e2728.

19. Netsch C, Knipper S, Bach T, et al. Impact of preoperative ureteral stenting on stone-free rates of ureteroscopy for

Cite this article as: Sung $\mathrm{LH}$, Cho DY. The role of preoperative ureteral stenting in retrograde intrarenal surgery in renal stone patients: a propensity score-matched study. Transl Androl Urol 2020;9(2):276-283. doi: 10.21037/tau.2020.03.09 nephroureterolithiasis: a matched-paired analysis of 286 patients. Urology 2012;80:1214-9.

20. Assimos D, Crisci A, Culkin D, et al. Preoperative JJ stent placement in ureteric and renal stone treatment: results from the Clinical Research Office of Endourological Society (CROES) ureteroscopy (URS) Global Study. BJU Int 2016;117:648-54.

21. Zelenko N, Coll D, Rosenfeld AT, et al. Normal ureter size on unenhanced helical CT. AJR Am J Roentgenol 2004;182:1039-41.

22. Kaplan AG, Lipkin ME, Scales CD Jr, et al. Use of ureteral access sheaths in ureteroscopy. Nat Rev Urol 2016;13:135-40.

23. Mogilevkin Y, Sofer M, Margel D, et al. Predicting an effective ureteral access sheath insertion: a bicenter prospective study. J Endourol 2014;28:1414-7.

24. Yang Y, Tang Y, Bai Y, et al. Preoperative double-J stent placement can improve the stone-free rate for patients undergoing ureteroscopic lithotripsy: a systematic review and meta-analysis. Urolithiasis 2018;46:493-9.

25. Shields JM, Bird VG, Graves R, et al. Impact of preoperative ureteral stenting on outcome of ureteroscopic treatment for urinary lithiasis. J Urol 2009;182:2768-74.

26. Perlmutter AE, Talug C, Tarry WF, et al. Impact of stone location on success rates of endoscopic lithotripsy for nephrolithiasis. Urology 2008;71:214-7. 